\title{
Antecedentes generales para la sustentabilidad de la producción hortícola en el valle de Azapa, Arica, Chile
}

\author{
General data for sustainability of horticultural production in \\ Azapa's Valley, Arica, Chile \\ Francisco González Vallejos, Alejandro Riquelme Garcés, \\ Pablo Contreras Luque y Pilar Mazuela ${ }^{1}$
}

\section{RESUMEN}

La región de Arica y Parinacota presenta condiciones climáticas excepcionales para el cultivo de hortalizas durante todo el año, siendo el principal proveedor de hortalizas para el país durante el invierno. La producción media regional supera significativamente la media nacional, con un alto valor comercial por la condición de "primor" que hace de la agricultura del valle de Azapa una actividad muy competitiva. Las principales ventajas para el cultivo de hortalizas son las condiciones naturales de suelo, clima y agua. Los principales problemas se vinculan al manejo del cultivo: técnicas de riego; control de plagas y enfermedades del suelo, y fertilización del cultivo, situación descrita hace más de 40 años y que se observa hasta hoy.

Palabras clave: contraestación, tomate, pimiento, eficiencia hídrica, emisión.

\begin{abstract}
The region of Arica and Parinacota has exceptional conditions for growing vegetables off season, being the main supplier of vegetables for the country during the winter. Average regional production is significantly major than the national average, with a high commercial value that makes horticulture of Azapa's Valley a very competitive activity. The main advantages for growing vegetables are the natural conditions of soil, climate and water. The main problems are related to crop management, especially irrigation techniques, pest and disease control and soil fertilization of the crop. This situation was described about 40 years ago and observed until today.
\end{abstract}

Key words: off-season, tomato, pepper, hydric efficiency, emission.

\section{Introducción}

Cuando se piensa en agricultura intensiva, esta se inicia con la mejora en los sistemas de regadío, la introducción de híbridos de altos rendimientos y la aplicación de fertilizantes. Una característica de los sistemas hortícolas intensivos es que han pasado de ser un sistema que busca mayor producción a uno que busca calidad principalmente porque se valoran aspectos como la salud de los productores, la salud de los consumidores y el cuidado del medio ambiente. Es común utilizar varios términos descriptivos o indicativos de una normativa o "etiqueta" que regula los procesos de producción y comercialización. Como ejemplo de estos términos tenemos: cultivo ecológico, cultivo biológico, agricultura sostenible, sustentable, agricultura no contaminante y amigable con el medio ambiente, producción controlada, producción integrada, etc. Todos ellos tienen de común un intento de racionalizar la producción con mayor o menor grado de exigencia y limitaciones en el sistema productivo.

Durante la década de los 70, la Junta de Adelanto de Arica generó un estudio agroeconómico para determinar la producción, organización, manejo y comercialización de la agricultura del Valle de Azapa. Los principales resultados de este estudio se describen a continuación y demuestran que la situación del valle no ha variado mucho en los últimos 43 años respecto de las condiciones naturales y manejo cultural que impide desarrollar todo su potencial productivo. Cabe indicar que la

\footnotetext{
1 Facultad de Ciencias Agronómicas, Universidad de Tarapacá, Arica, Chile. Autor por correspondencia: pmazuela@uta.cl
} 
productividad ha mejorado significativamente, cuadruplicando los rendimientos medios en torno a las de $30 \mathrm{t} \mathrm{ha}^{-1}$ de principios de los años 70 . Sin embargo, la introducción de sistemas de riego más eficientes, pasando del riego por caracol a riego por goteo, ha sido insuficiente para mejorar la eficiencia hídrica y la sustentabilidad del recurso hídrico, principalmente debido a que las tecnologías que se adoptan son transferidas como en el lugar de origen, sin considerar, que las condiciones de clima, agua y suelo son diferentes.

\section{Condiciones naturales para la producción de hortalizas}

El valle de Azapa cuenta con condiciones de clima, suelo y agua que la hacen apta para el cultivo de hortalizas durante todo el año. Este valle se sitúa en las coordenadas 18²31'2'S 70¹1'31'O. El ancho del valle es variable y fluctúa entre $700 \mathrm{~m}$ y $2.200 \mathrm{~m}$; con una pendiente de $1,7 \%$ y un largo aproximado de $58 \mathrm{~km}$.

La intensificación de la agricultura ha generado una mayor competencia por los recursos naturales, especialmente suelo y agua. En pocos años el costo de la tierra agrícola se ha cuadruplicado, lo que obliga a mejorar los sistemas productivos para hacer sostenible esta actividad.

\section{Clima}

Predominan las condiciones de clima de desierto costero con nubosidad abundante, ausencia de heladas, vientos moderados, alta humedad relativa y alta radiación solar directa, durante todo el año. Si comparamos las temperaturas medias de principios de la década de los 70 y las actuales (Tabla 1), se observa que ha bajado la temperatura media anual, principalmente por una disminución en las

Tabla 1. Evolución de la temperatura $\left({ }^{\circ} \mathrm{C}\right)$ máxima media y mínima media, Arica año 1971 y 2008.

\begin{tabular}{lll}
\hline & $1971^{1}$ & $2008^{2}$ \\
\hline Temperatura media anual & 19,3 & 18 \\
Temperatura máxima media & 21,8 & 23,6 \\
Temperatura mínima media & 15,2 & 13,8 \\
Diferencia & 6,6 & 9,8 \\
\hline
\end{tabular}

Fuente: ${ }^{1}$ Espina (1971); ${ }^{2}$ Torres y Acevedo (2008). temperaturas mínimas medias. Sin embargo, las máximas medias han aumentado y la diferencia entre mínimas y máximas medias también ha aumentado.

Esta tendencia sugiere que la propensión al aumento de la superficie cultivada bajo malla no solo permite mantener en mejores condiciones fitosanitarias el cultivo, sino, además, permite mantener al interior del invernadero temperaturas mínimas más altas y máximas más bajas que los cultivos al aire libre.

\section{Agua}

El agua que dispone Arica y el valle de Azapa proviene de la precordillera al oriente de la sierra de Huaylillas. Esta agua llega en parte por el canal Lauca y sobre todo por aguas subterráneas. Sin embargo, es el río San José, o Azapa, el principal recurso hidrológico de este valle, sus aguas provienen de las precipitaciones de las quebradas de Seca, Chusmiza y Ticnamar. El agua de este río corre superficialmente durante los años lluviosos y subterráneamente en los años secos, apareciendo por vertientes, norias o pozos. Desde las fuentes primarias del agua, estas se infiltran en el terreno aluvial. Por las condiciones geológicas y climáticas, el agua que percola a la napa subterránea aumenta su concentración de sales a lo largo de su curso. Espina (1971) indica que el agua de la cuenca del valle de Azapa no solo está destinada a las explotaciones agrícolas, sino también al consumo humano de la creciente población de Arica y sus industrias allí instaladas. Como se observa, hace más de cuatro décadas ya existía preocupación por los altos requerimientos hídricos de la agricultura y por las necesidades de la población de una ciudad que crecía y se industrializaba.

La sobreexplotación del acuífero del San José está afectando la disponibilidad y calidad de agua debido a: 1) habilitación de suelos; 2) ineficiencia en las técnicas de riego y, 3) sobrefertilización. El acuífero del río San José, además, es la fuente de agua potable para la ciudad de Arica. Según la Dirección General de Aguas (DGA), la explotación del acuífero de Azapa está sobre su capacidad sustentable, del orden de $700 \mathrm{~L} \mathrm{seg}^{-1}$, pues los derechos de aprovechamiento de agua superan los $3000 \mathrm{~L} \mathrm{seg}^{-1}$ y la explotación real es de $1000 \mathrm{~L} \mathrm{seg}^{-1}$.

En la Tabla 2 se observa el efecto de la técnica de cultivo en la producción media y la eficiencia en el uso del agua al aire libre y bajo protección con 
Tabla 2. Producción media $\left(\mathrm{kg} \mathrm{m}^{-2}\right)$, eficiencia en el uso del agua ( $\mathrm{kg}$ producidos $\mathrm{m}^{-3}$ agua aportado) y emisión de iones al medio ambiente ( $\mathrm{g}$ emitidos por $\mathrm{kg}_{\text {producido }}{ }^{-1}$ ), según cultivo y sistema de cultivo.

\begin{tabular}{lrrr}
\hline & \multicolumn{2}{c}{ Tomate } & \\
\cline { 2 - 3 } & Aire libre & Malla antiáfido & Plástico \\
\hline Producción $^{1}$ & 7,6 & 20,6 & 11,0 \\
Eficiencia en uso del agua $^{1}$ & 12,5 & 25,6 & 13,6 \\
Emisión iones $^{2}$ & 79,61 & 42,82 & 73 \\
\hline
\end{tabular}

Fuente: ${ }^{1}$ Riquelme et al., 2013; ${ }^{2}$ Elaboración propia a partir de los datos de ${ }^{1}$ Riquelme et al., 2013.

malla antiáfido. La producción media de tomate en cultivo protegido se triplica y la eficiencia en el uso del agua se duplica, respecto del cultivo al aire libre. La mayor producción de tomate bajo malla se debe a las mejores condiciones de cultivo que evita el envejecimiento prematuro de la planta y aumenta el calendario de producción comercial, por lo tanto, aumenta la producción total por superficie cultivada. Para el caso del pimiento solo se consideró el cultivo bajo plástico.

\section{Suelos}

Los suelos son aluviales con una marcada estratificación. Las texturas más comunes son: a) en la superficie, la franco-arcillosa fina a franca $y, b)$ en profundidad, se alternan las estratas de texturas arenosas y, ocasionalmente, arcillosas. Los suelos poseen una buena saturación de bases, son calcáreos, de reacción moderadamente alcalina y de buena porosidad. Sin embargo, son de poca profundidad y con un exceso de pedregosidad. A consecuencia de la calidad del agua de riego y su manejo, se describe que los suelos poseen un cierto tenor salino que limita su potencial. No obstante lo anterior, pese a que los suelos y las aguas presentan moderados problemas, el estudio liderado por Espina (1971) indica que la causa fundamental de la baja productividad de ellos se debe a factores de manejo, siendo los más importantes: 1) manejo del agua de riego (métodos de riego); 2) control fitosanitario del suelo y, 3) aplicación de fertilizantes.

En este último punto se hace especial énfasis a que hay un desconocimiento generalizado de las normas técnicas elementales de dosificación, situación que persiste hasta hoy. Por ejemplo, el agua de riego del canal Lauca no aporta nitratos ni fosfatos. Sin embargo, en la Tabla 3 se observa cómo la sobrefertilización genera la emisión de contaminantes al medio ambiente en el drenaje del agua no consumida por una planta.

\section{Importancia de la producción de hortalizas en el valle de Azapa}

El valle de Azapa es el gran productor de hortalizas que abastece a todo el país durante el invierno. En diciembre del 2004 el Servicio Agrícola y Ganadero (SAG) de Chile declaró a la provincia

Tabla 3. Emisión diaria (me $\left.\mathrm{L}^{-1} \mathrm{~m}^{-2} \mathrm{~d}^{-1}\right)$ y total acumulado en 100 días $\left(\mathrm{g} \mathrm{m}^{-2}\right)$ de nitratos y fosfatos, para un cultivo de tomate, según sustrato

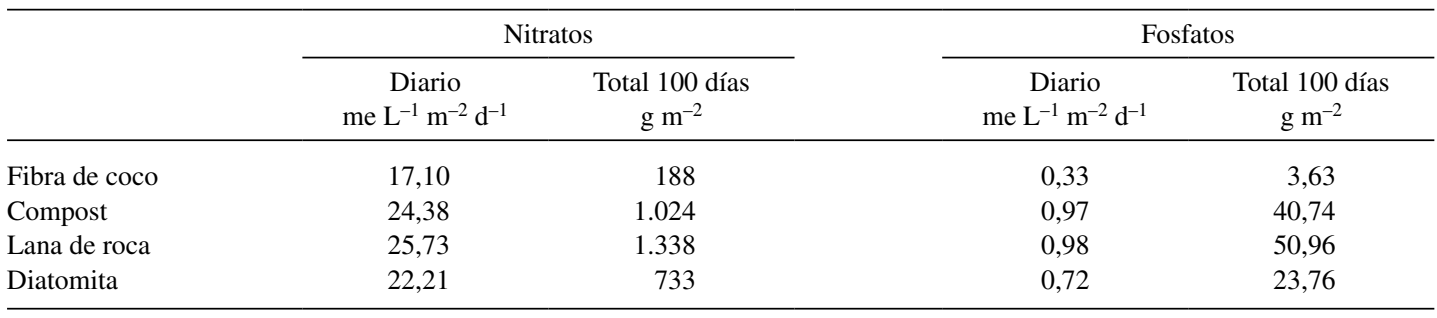

Fuente: Elaboración propia en base a parámetros de fertirriego. 
de Arica como un lugar libre de la mosca de la fruta (Ceratitis capitata) y el principal efecto de esta medida fue la diversificación de la agricultura en los valles costeros de la comuna de Arica, caracterizada, hasta entonces, por el monocultivo del tomate, única hortaliza autorizada para salir fuera de la comuna previo tratamiento cuarentenario con bromuro de metilo. Al comparar la evolución de la superficie cultivada cuando existían restricciones por la mosca de la fruta, respecto de la superficie cultivada una vez eliminada la mosca de la fruta (Tabla 4), se observa que el total destinado a hortalizas aumentó
50\% en un período de 10 años, con un importante incremento de la superficie cultivada con pimiento $\mathrm{y}$ tomate.

Al comparar los rendimientos medios de las principales hortalizas cultivadas en la región, estas superan significativamente las medias nacionales, como se observa en la Tabla 5. Las hortalizas del valle de Azapa tienen un alto valor comercial debido a la productividad alcanzada y a la condición de hortalizas de "primor" que hace de la horticultura del valle de Azapa una actividad muy competitiva a nivel regional y nacional.

Tabla 4. Evolución de la superficie cultivada (há) de hortalizas en la comuna de Arica, y rendimientos medios $\left(\mathrm{Kg} \mathrm{m}^{-2}\right)$ por región y nacional

\begin{tabular}{|c|c|c|c|c|}
\hline \multirow{2}{*}{ Especie } & \multicolumn{2}{|c|}{ há } & \multicolumn{2}{|c|}{${ }^{3} \mathrm{Kg} \mathrm{m}^{-2}$} \\
\hline & ${ }^{1} 1997$ & ${ }^{2} 2007$ & XV Región & Nacional \\
\hline Ají & 4,5 & 13,9 & s.i. & s.i. \\
\hline Berenjena & 1,7 & 9,8 & s.i. & s.i. \\
\hline Lechuga* & 18,5 & 54,9 & 2,52 & 4,29 \\
\hline Pepino & 20,3 & 37,3 & s.i. & s.i. \\
\hline Pimiento & 85,8 & 138,4 & 4,65 & 3,70 \\
\hline Poroto verde & 308,7 & 171,5 & 0,98 & 0,84 \\
\hline Tomate & 448,5 & 840,1 & 11,29 & 7,11 \\
\hline Zapallo italiano & 34,1 & 105,6 & s.i. & s.i. \\
\hline Total & 922,1 & 1371,5 & & \\
\hline
\end{tabular}

Fuente: ${ }^{1}$ Instituto Nacional de Estadísticas, $1997 ;{ }^{2}$ Instituto Nacional de Estadísticas, 2008; ${ }^{3}$ Instituto Nacional de Estadísticas, 2010. s.i.: sin información.

* Unidades $\mathrm{m}^{-2}$.

Tabla 5. Superficie (ha), total nacional y regional; rendimiento $\left(\mathrm{kg} \mathrm{ha}^{-1}\right)$, media nacional y media regional para cultivo de tomate y pimiento.

\begin{tabular}{|c|c|c|c|c|}
\hline \multirow{2}{*}{ Región } & \multicolumn{2}{|c|}{ Tomate } & \multicolumn{2}{|c|}{ Pimiento } \\
\hline & ${ }^{1}$ há & ${ }^{2} \mathrm{~kg} \mathrm{ha}^{-1}$ & ${ }^{1}$ há & ${ }^{2} \mathrm{~kg} \mathrm{ha}^{-1}$ \\
\hline Nacional & 6.309 & 71.100 & 1.567 & 36.960 \\
\hline XV & 840 & 112.860 & 138 & 46.500 \\
\hline III & 212 & 61.570 & 22 & 42.470 \\
\hline IV & 358 & 30.800 & 601 & 32.450 \\
\hline $\mathrm{V}$ & 1.179 & 94.350 & 127 & 35.020 \\
\hline VI & 1.062 & 58.730 & 333 & 42.160 \\
\hline VII & 938 & 68.870 & 116 & 45.900 \\
\hline VIII & 467 & 49.710 & 1 & 33.980 \\
\hline $\mathrm{RM}$ & 1.080 & 61.870 & 227 & 29.320 \\
\hline
\end{tabular}

Fuente: ${ }^{1}$ INE, 2008, ${ }^{2}$ INE, 2010. 


\section{Conclusión}

El valle de Azapa tiene condiciones naturales que permiten la producción de hortalizas de gran valor comercial, tanto por los altos rendimientos medios alcanzados como por su condición de primor que permite abastecer de estos productos a todo el país durante el invierno. Sin embargo, es necesario mejorar algunos aspectos de manejo del cultivo, especialmente vinculado a las técnicas del riego y fertilización para dar sustentabilidad a esta actividad en el valle de Azapa.

\section{Agradecimientos}

Este trabajo ha sido financiado por el proyecto FONDEF D10R1026.

\section{Literatura Citada}

Espina, L.

1971. Estudio agroeconómico del valle de Azapa. Junta de Adelanto de Arica-Universidad de Chile, Santiago de Chile. $155 \mathrm{p}$.

Instituto Nacional de Estadísticas.

2010. Información Hortícola. Publicación Especial 2008-2009.

INE, Ediciones, Santiago de Chile, 128 p.

Instituto Nacional de Estadísticas.

1999. VI Censo Nacional Agropecuario, Región de Tarapacá. Edición Rossana Espinosa Peralta. Iquique, Chile. 473 p.
Instituto Nacional de Estadísticas.

2008. VII Censo Agropecuario y Forestal 2006-2007. Resultados preliminares. INE, Ediciones, Santiago de Chile, 444 p.

Riquelme, A.; González, F.; Contreras, P.; Mazuela, P. 2013. Manejo del cultivo de hortalizas y su efecto en la sustentabilidad de un valle costero del desierto de Atacama, Chile. Idesia 31 (3): 113-117.

Torres, A. y Acevedo, E.

2008. El problema de salinidad en los recursos suelo y agua que afectan el riego en los valles de Lluta y Azapa en el norte de Chile. Idesia 26 (3): 31-44. 
